\title{
Workflow of Fracture Prediction Using Curvature-Related Attributes and a Case Study
}

\author{
Chang Deshuang $^{1}$, Chen Zhigang ${ }^{1}{ }^{*}$, Xu Jianguo ${ }^{2}$, Han Yuchun ${ }^{1}$, Sun Xing $^{1}$, Guo Jianming ${ }^{1}$ \\ ${ }^{1}$ Geology Research Center of Research Institute, BGP Inc., China National Petroleum Corporation, Zhuozhou, China \\ ${ }^{2}$ China National Oil and Gas Exploration and Development Corporation, Beijing, China
}

Email address:

Chenzhigang@cnpc.com.cn (Chen Zhigang)

${ }^{*}$ Corresponding author

\section{To cite this article:}

Chang Deshuang, Chen Zhigang, Xu Jianguo, Han Yuchun, Sun Xing, Guo Jianming. Workflow of Fracture Prediction Using Curvature-Related Attributes and a Case Study. American Journal of Physics and Applications. Vol. 9, No. 5, 2021, pp. 127-132. doi: 10.11648/j.ajpa.20210905.15

Received: July 26, 2021; Accepted: August 4, 2021; Published: October 28, 2021

\begin{abstract}
Fracture is a kind of important reservoir in petroleum exploration, which usually exist in the carbonate rock or igneous rock. However, it is always difficult to predict the fracture with the seismic data. In this paper, based on curvature attributes, we develop a workflow for the prediction of fractured zone, fracture orientation, and open fractures. We begin with curvature calculation to predict fractured reservoirs and then calculate rose diagrams using curvature data. Fracture orientation is established by comparing the rose diagrams from imaging logs and the analogues from curvature data. We identify two principal orientations and calculate the azimuth intensity in these two directions using the curvature data. As per the crossplots of azimuth intensity in two directions and productivity, the azimuth with good correlation is the open azimuth of fractures. We apply this method to a Kazakhstan oilfield $\mathrm{K}$ and predict fractured-vuggy reservoirs in the eastern field and fractured reservoirs in the western field. In accordance with the prediction, there are two groups of fractures, one in a northeast direction and the other in a northwest direction. NE fractures are open in the northern field, and NW fractures are open in the southern field. We suggest two sites for well drilling, which obtain economic oil flow.
\end{abstract}

Keywords: Fractured Reservoir, Anisotropy, Curvature, Azimuth Intensity, Rose Diagram

\section{Introduction}

The region of interest is an oilfield $\mathrm{K}$ in Basin $\mathrm{N}$, Kazakhstan with buried-hill carbonate rocks as one of the major targets. There are fractured and dissolved vuggy reservoirs with strong heterogeneity. As per drilling data, there is a positive correlation between reservoir properties and hydrocarbon production. Dissolved vuggy reservoirs were successfully predicted using impedance inversion due to large impedance contrast between reservoirs and wall rocks and consequent strong geophysical responses. But it was hard to predict fractured reservoirs using routine seismic attributes and impedance inversion owing to their faint geophysical responses. Thus, how to predict fractured reservoirs is a task of top priority.

Despite the complicated relationships between fractures and curvatures, preceding studies showed a correlation between open fractures and intense tectonic deformation, e.g. open fractures related to flexure in tight sandstone [1], natural fractures directly related to curvatures [2], high production related to the structural lows indicated by the most negative principal curvature in the Woodford Shale, the Akama Basin [3], and natural fractures in the Frontier Formation at the outcrops in the northern Rocky Mountains highly correlated with the palaeostress (calculated through paleostructure restoration) and the curvatures nowadays [4]. The studies of the relations between fluids and seismic curvatures began in 2009. A study of the Dickman oilfield in Kansas, the US showed that there was a close correlation between fluids and the distance from the well to the nearest fractures on the curvature map. In other words, water production decreased with the distance from the well to the nearest fractures in a northwest direction, which means that NW fractures are open; oil and gas production increased with the distance from the well to the nearest fractures in a northeast direction, which 
means that NE fractures are closed. These conclusions were confirmed by the results of horizontal well drilling [5]. Thus, more efforts have focused on fracture prediction using curvature attributes [6-8], most of which were limited to the prediction of fractured zones. Based on the attributes derived from curvature (curvature attributes), we develop a workflow for the prediction of fractured zone, fracture orientation, and open fractures.

\section{Workflow}

The workflow included 3 steps, i.e. predicting fractured zones using routine curvature attribute volumes, predicting fracture orientations using the rose diagrams derived from curvature attributes, and predicting open fractures using the azimuth intensity derived from curvature, as shown in Figure 1. Post-stack seismic data and GeoEast developed by CNPC BGP were used to accomplish the prediction. The whole process was detailed as follows.

(1) Fractured zones were predicted using routine curvature attribute volumes, which were extracted based on dip scanning. Multi-window dip scanning was performed first to calculate the dip angle in the inline and crossline directions. Major parameters included 9 time windows of $12 \mathrm{~ms}$ for each, the maximum angle of $40^{\circ}$ for scanning, and the step of $5^{\circ}$. Curvatures, e.g. maximum curvature, minimum curvature, maximum positive curvature, and maximum negative curvature, were then calculated [9-11].

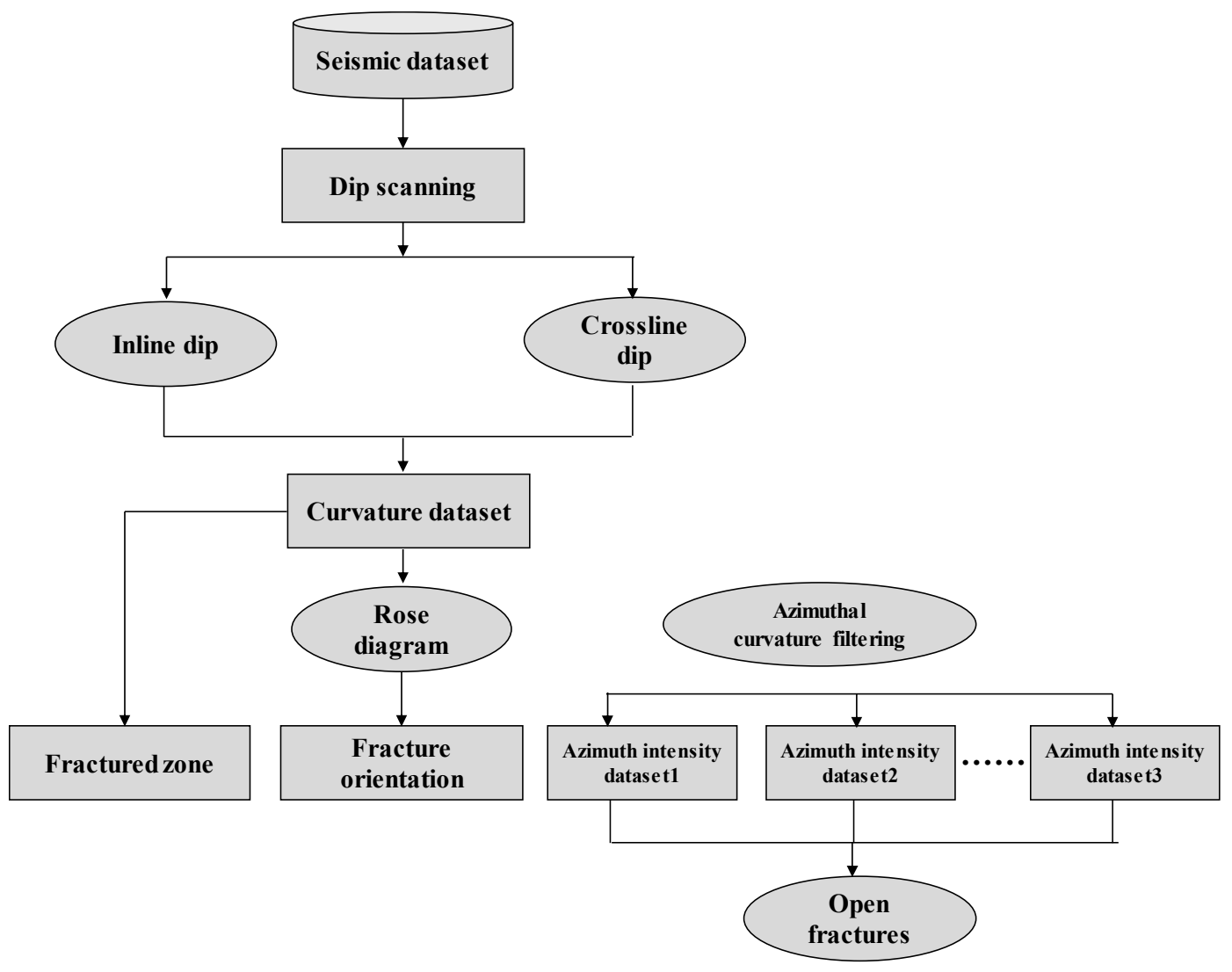

Figure 1. Fracture prediction workflow.

(2) Rose diagrams were obtained to establish fracture orientations. Rose diagrams are generally derived from imaging logging to indicate fracture orientation. The direction of vector pointing to the north is defined as $0 \mathrm{o}$, and the included angle between the fracture strike at a point and the north is defined as the azimuth of fracture [12]. Before calculating rose diagram using curvature, we estimated fracture orientation using maximum positive curvature $k 1$, maximum negative curvature $k 2$, maximum curvature, and minimum curvature [13]. For $|k 2|>|k 1|$, the fracture orientation was equal to the azimuth of maximum curvature; otherwise the fracture orientation was equal to the azimuth of minimum curvature. After that, we used rose diagrams to show the azimuths (Figure 2b). The frequency of fractures occurring in each direction was normalized and plotted on the rose diagram to indicate fracture distribution in the region of interest [14]. Major parameters included the diameter of rose diagram and number of leaves; the former was directly related to the size of the region of interest and the latter was generally set to range 1-15.

(3) Open fractures were predicted using azimuth intensity. Nissen et al. investigated the relation between fluids and curvature volumes in the study of the Dickman oilfield in Kansas, the US [5]. In accordance with the crossplot of fracture azimuth and water or oil production, they found a correlation between fluids and the distance from the well to the nearest fractures and concluded that fracture azimuth with good correlation is open fracture azimuth. Guo [13] presented 
two deficiencies in Nissen's study; one was the error resulting from man-made data and the other was fluids limited within the area close to the well site. Based on Nissen's idea, Guo assumed that fluids are related to all fractures, instead of those fractures close to the well site, and fracture density is proportional to curvature value. Based on these two assumptions, Guo developed the attribute of azimuth intensity through azimuthal curvature filtering to obtain azimuthal curvature, followed by the convolution of azimuthal subdata and $1 / r$, where $r$ is the distance between a specific point and nearby fractures. This method was based on the statistical correlation presented by Nissen et al. in 2009. That is the Green function describing fluid flow in a horizontal layer penetrated by a vertical well or across a fault surface could be simplified as $1 / \mathrm{r}$, where $\mathrm{r}$ is the radial distance and $\mathrm{r}=(\mathrm{x} 2+\mathrm{y} 2) 1 / 2 ; 1 / \mathrm{r}$ could be taken as the pulse response shown in Figure 2d. In view of the positive correlation between fluids and open fractures, the azimuth intensity could be used to predict open fractures.

\section{A Case Study}

Oilfield $\mathrm{K}$ is an anticline with its major axis in a nearly north-south direction (Figure 2a). There are fractured and dissolved vuggy reservoirs with strong heterogeneity in carbonate buried hills [15]. Reservoir distribution is dependent on lithologies. Limestone formations with small shale content mainly occur in lower buried hills; argillaceous limestones or calcareous mudstones with increased shale content turn up in upper buried hills. The northeast part was topographically higher than the middle and southwest parts and uplifted at the early stage. As a result, the formations with high shale content were denuded in the upper section, where there are mainly dissolved vuggy reservoirs and some fractured reservoirs. Limestones with high shale content have been well preserved in the middle and southwest parts, where there are mainly fractured reservoirs and some dissolved vuggy reservoirs (Figure 2b). There is a distinct correlation between reservoir properties and hydrocarbon production. The northeast part exhibits better reservoir properties, where hydrocarbon production is higher than other areas (Figure 2c). In accordance with the crossplot of log data, impedance could be used to differentiate between dissolved vuggy reservoirs and wall rocks; thus, we could use impedance inversion for prediction. This method is not feasible for the prediction of fractured reservoirs due to small impedance contrast; thus, we used curvature attributes to predict fractures.
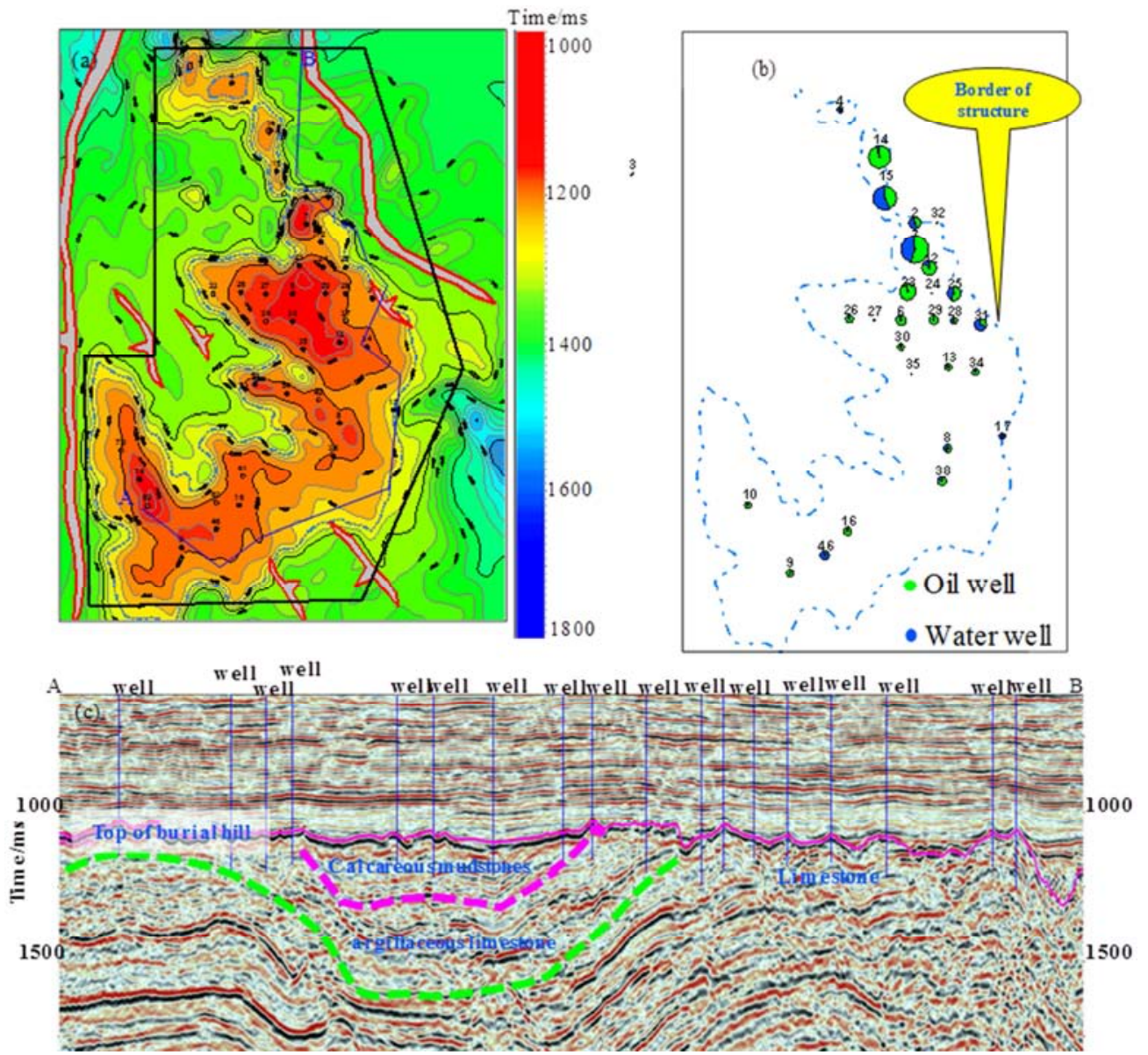

(a) Structure map; (b) Fluid production in the region of interest; (c) Seismic section with its location shown as a blue line in Figure (a)

Figure 2. Structure map, fluid production, and a seismic section acorss the region of interest. 


\subsection{Fractured Zones Predicted Using Curvatures}

Many wells were drilled with fractured reservoirs, and oil layers mainly turn up at the top of buried hills. Figure 2c shows irregular surface at the top of buried hills, and considerable wells were drilled at xiphoid anticlines. This means that maximum positive curvature may be used to predict fractures. Figure 3 shows prediction results. Figure 3a shows a curvature slice, in which fractured or fractured-vuggy zones are plotted in black. Sparse black lines in the most region correspond to fractures, which have been confirmed by well drilling (plotted in green); dense black lines in the northeast correspond to fractured-vuggy zones, which may be predicted through impedance inversion. Figure $3 \mathrm{~b}$ shows the thickness of fractured-vuggy reservoirs obtained using impedance inversion. Thick fractured-vuggy reservoirs in the northeast are reconciled with the curvature attribute; this means that curvature may be used to predict fractures and vugs in addition to fractures. There is also a fractured-vuggy zone in the southeast corner. Figure $3 \mathrm{c}$ shows a seismic section with its location plotted as a light blue line in Figure 3a; the numbers in these two figures correspond to each other. Black anomalies correspond to xiphoid anticlinal reflections in the seismic section; fractures at 3, 4, 5, and 6 have been confirmed by well drilling. As per drilling data, the coincidence rate of curvature prediction was above $90 \%$ in the region of interest.
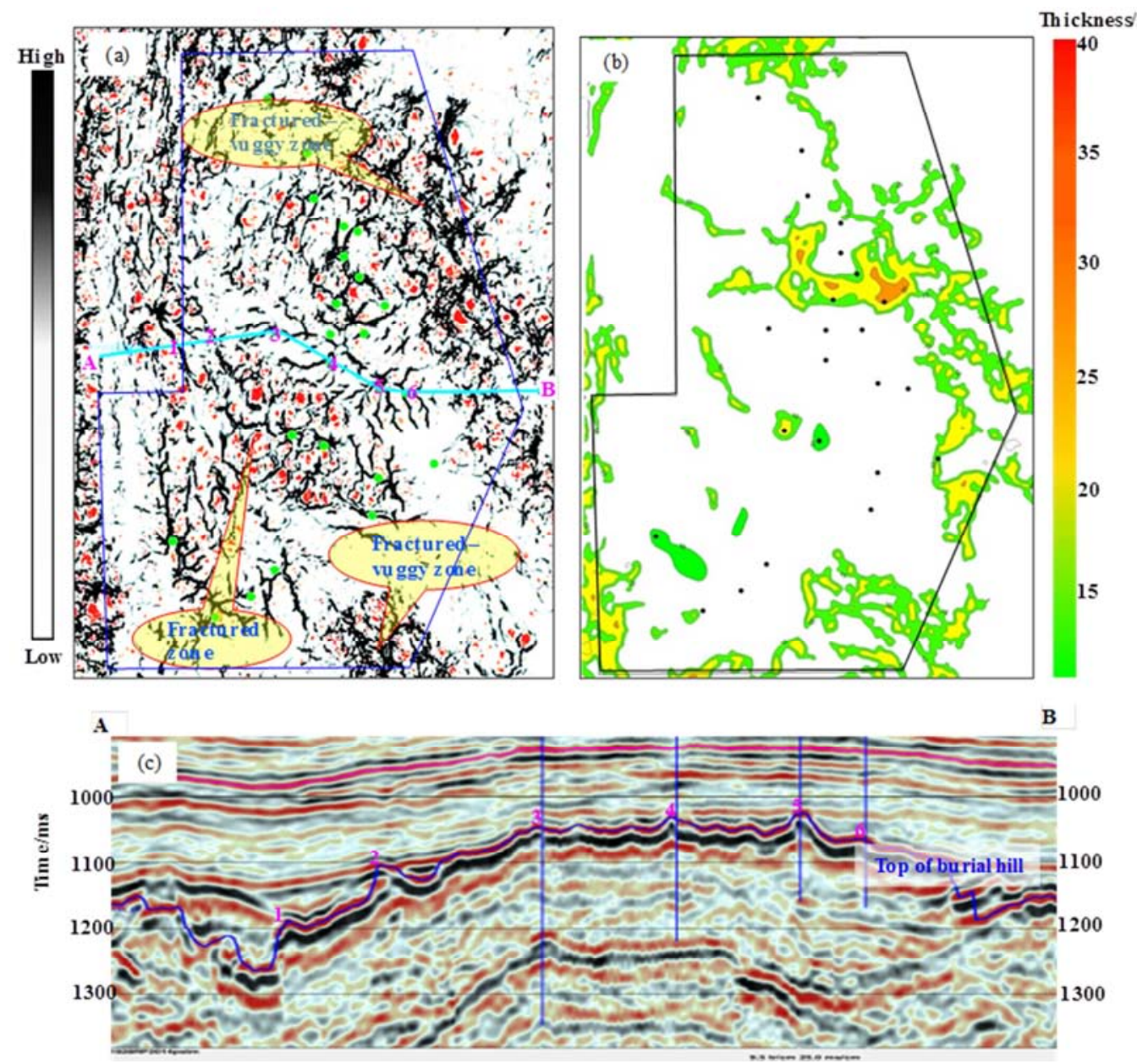

Figure 3. Results of fracture prediction.

(a) Horizon slice of curvature at the top of buried hills; (b) Fractured-vuggy reservoir thickness obtained using impedance; (c) Seismic section with its location shown as a blue line in Figure (a)

Note: The blue polyline in (a) or black polyline in (b) is the project boundary.

\subsection{Fracture Orientations Predicted Using Rose Diagrams}

Figure 4a shows seismic predicted rose diagrams. Each square is a cell with the same fracture orientation. The intersection point of several cells will be affected by these cells, i.e. fractures in different orientations. In general, there are two groups of fractures, one in a northeast direction and the other in a northwest direction. Figure $4 \mathrm{~b}$ shows the rose diagrams obtained from imaging logging, which correspond to the analogues at well sites in Figure 4a. We compared these two figures and concluded that the coincidence rate may be above $80 \%$. 

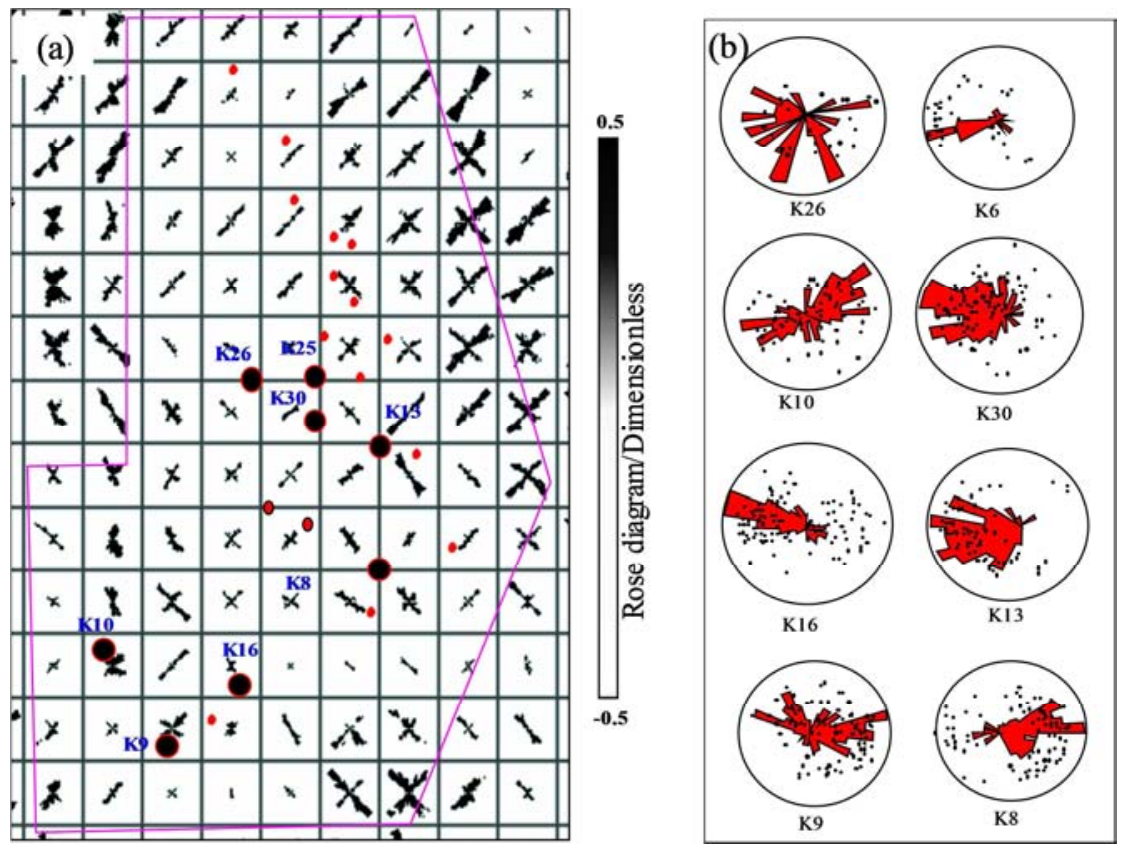

Figure 4. Rose diagrams obtained from seismic prediction and imaging logging.

(a) Rose diagrams obtained from seismic prediction; (b) Rose diagrams obtained from imaging logging Note: The pink polyline in (a) shows the boundary of the project.
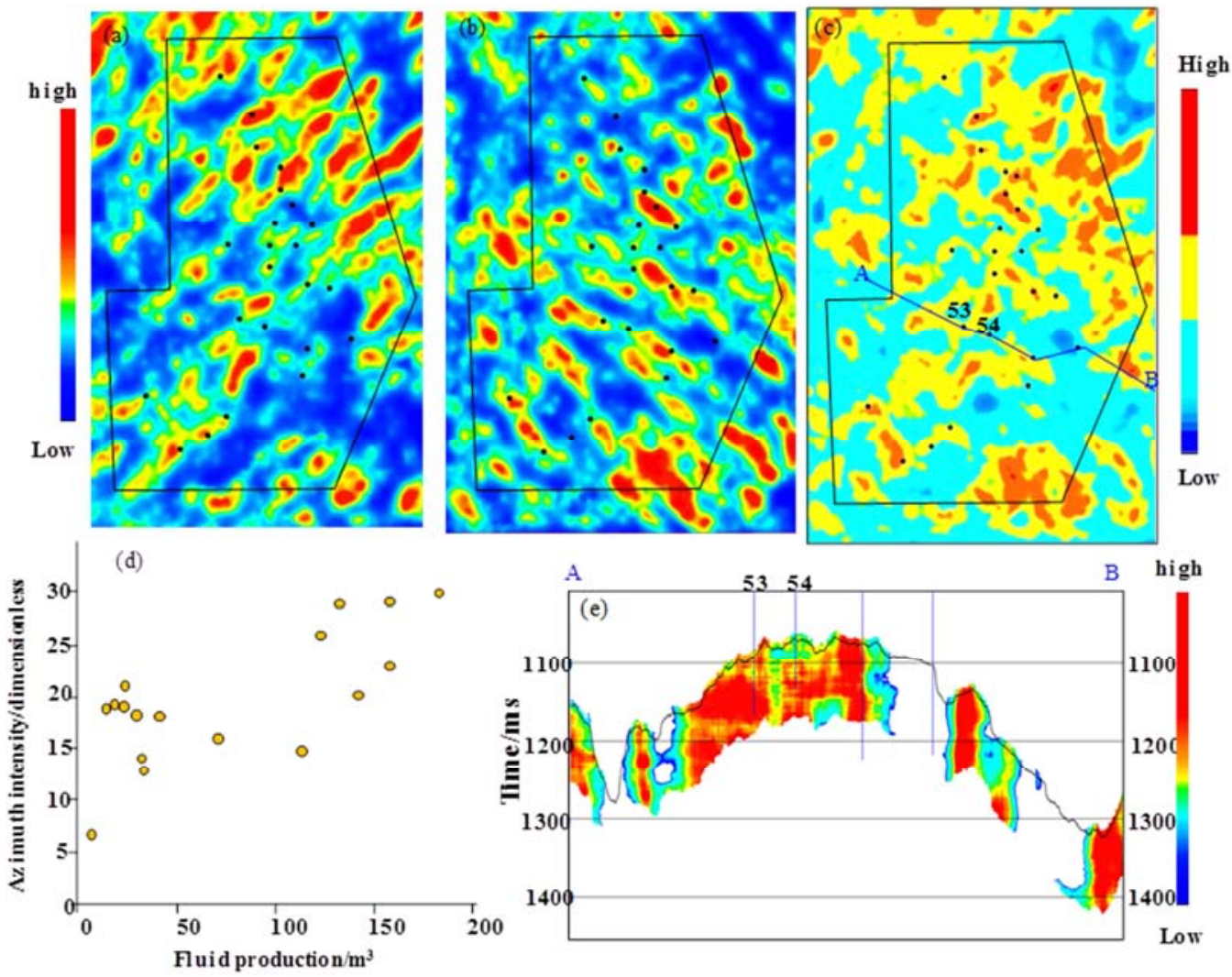

Figure 5. Open fractures predicted using azimuth intensity.

(a) Azimuth intensity in a northeast direction (high value in red and yellow for NE open fractures); (b) Azimuth intensity in a northwest direction (high value in red and yellow for NW open fractures); (c) NE and NW azimuth intensity fusion (high value in red and yellow for high-graded fractured reservoirs); (d) Crossplot of azimuth intensity fusion and fluid production; (e) Section of azimuth intensity fusion Note: The black lines in (a), (b) and (c) are the project boundary. 


\subsection{Open Fractures Predicted Using Azimuth Intensity}

Azimuth intensity may be decomposed in several directions. As shown in Figure 4, two conjugate directions, northeast and northwest, were predicted. Thus, azimuth intensity was decomposed into two components, one in a northeast direction (Figure 5a) and the other in a northwest direction (Figure 5b). Large azimuth intensity indicates open fractures; thus, Figure 5a shows that NE fractures are open in the northeast part, and Figure $5 \mathrm{~b}$ shows that NW fractures are open in the middle and south parts. Figure $5 \mathrm{c}$ shows a fusion attribute of those in Figures $5 \mathrm{a}$ and $5 \mathrm{~b}$, which comprehensively indicates the occurrence of open fractures. Red and yellow colors indicate the areas with high-graded fractured reservoirs, especially in the northeast; this agrees with the trend in Figure $2 \mathrm{~b}$. As per the crossplot (Figure $5 \mathrm{~d}$ ), there is a positive correlation between the attribute and fluid production at the well sites shown in Figure $5 \mathrm{c}$; this means that the degree of opening is proportional to fluid production. In accordance with fracture prediction and structures, we proposed two sites, 53 and 54, for well drilling (with the section shown in Figure 5e and location shown in Figure 5c). Well 53 yielded daily oil of $326 \mathrm{~m}^{3}$ in oil testing with the choke of $7 \mathrm{~mm}$; Well 54 has not been tested.

\section{Conclusions}

1) As a kind of important reservoir in the petroleum exploration, fracture can be predicted effectively with the 3D seismic data. We proposed a workflow for the prediction of fractured zone, fracture orientation, and open fractures. We calculated curvatures using seismic data to predict fractured reservoirs and then generated rose diagrams using curvature data to predict fracture orientations. The azimuth of open fractures was predicted using azimuth intensity.

2) We predicted fractured reservoirs in the west part of oilfield $\mathrm{K}$ using curvature attributes. Two groups of fractures, one in a northeast direction and the other in a northwest direction, were predicted. NE fractures were predicted to be open in the north, and NW fractures were predicted to be open in the south. The coincidence rate was above $80 \%$. Two wells suggested obtained economic oil flow.

\section{References}

[1] Hart, B. S., R. A. Pearson, and G. C. Rawling. 3D seismic horizon-based approaches to fractures warm sweet spot definition in tight-gas reservoirs [J]. The Leading Edge, 2002, 21: $28-35$.
[2] Narhari, S. R., A. L. Al-Kandari, V. K. Kidambi, et al. Understanding fractures through seismic data: North Kuwait case study [C]. 2009, 79th Annual International Meeting, SEG, Expanded Abstracts: 547-550.

[3] Guo, Y., K. Zhang, K. J. Marfurt. Seismic attribute illumination of Woodford Shale faults and fractures, Arkoma Basin [C]. 2010, 80th Annual International Meeting, SEG, Expanded Abstracts: 1372-1376.

[4] Hennings, P. H., J. E. Olson, et al. Combining outcrop data and three-dimensional structural models to characterize fractured reservoirs: a example from Wyoming [J]. AAPG Bulletin, 2000, 84: 830-849.

[5] Nissen, Carr, K. J. Marfurt, et, al. Using 3D seismic volumetric curvature attributes to identify fracture trends in a depleted Mississippian carbonate reservoir: Implications for assessing candidates for $\mathrm{CO}_{2}$ sequestration $[\mathrm{J}]$. AAPG Studies in Geology, 2009, 59: 297-319.

[6] Cui L J, He Y N, Wang J X et al. Application of seismic curvature based on horizon to carbonate fault prediction: An example of an area in Tabei, Tarim Basin [J]. Lithologic Reservoirs, 2012, 24 (1): 92-96.

[7] Wang H J, Wang L L, Wan X M et al. Fracture Prediction of Low Permeability Reservoirs of the Mesozoic in North Yellow Sea Basin [J]. Xinjiang Petroleum Geology, 2014, 35 (3): 268-272.

[8] Sheng X L. Minor Fault Identification Based on 3D Seismic Curvature [J]. Coal Geology of China, 2018, 30 (S1): (109-117).

[9] Roberts, A. Curvature attributes and their application to interpreted horizons [J]. First Break, 2001, 19: 85-99.

[10] Bergbauer, S. T. Mukerji, P. Hennings. Improving curvature analyses of deformed horizons using scale-dependent filtering techniques [J]. AAPG Bulletin, 2003, 87: 1255-1272.

[11] Al-Dossary, K. J. Marfurt. 3-D volumetric multispectral estimates of reflector curvature and rotation $[\mathrm{J}]$. Geophysics, 2006, 71: 41-51.

[12] Zhang W, Yan J G, Zhou H S. The application of seismic rose in the analysis of fracture $[\mathrm{J}]$. Periodical agency of Yangze university, 2012, 9 (12): 61-63.

[13] Guo Y X, Zhang K, Kurt J. Marfurt. Quantitative correlation of fluid flow to curvature lineaments [C], SEG Las Vegas Annual meeting, 2012, Abstracts: 501-506.

[14] Zhang W. Seismic Rose Diagrams Analysis for Fracture Prediction and Tectonic Stress [D]. Chengdu: Chengdu University of Technology, 2013.

[15] Kong X Y, Yin J Y, Zhang F Q. Oil gas geological features and its exploration potential in South Turgay Basin, Kazakhstan [J]. Lithologic Reservoirs, 2007, 9 (3): 48-54. 